\title{
Clinical Features of Japanese Patients with Ocular Inflammation and Their Surgical Procedures Over the Course of 20 Years
}

This article was published in the following Dove Press journal: Clinical Ophthalmology

\author{
Nanae Takai \\ Takatoshi Kobayashi \\ Teruyo Kida \\ Tsunehiko Ikeda \\ Department of Ophthalmology, Osaka \\ Medical College, Takatsuki, Japan
}

Correspondence: Teruyo Kida Email opt038@osaka-med.ac.jp
Purpose: To review clinical characteristics of ocular inflammation patients in Osaka, Japan, over 20 years and investigate the efficacy of required surgeries.

Methods: We conducted a retrospective study involving 2730 eyes of 1815 patients with ocular inflammation examined at the Osaka Medical College Hospital from April 1999 to March 2019.

Results: Patients comprised 843 males and 972 females, with a mean age of $56.3 \pm 18.5$ years. Anterior uveitis, such as scleritis, acute anterior uveitis, and herpes iritis, was the most common anatomical classification $(51.2 \%)$, followed by panuveitis $(37.2 \%)$, posterior uveitis (9.4\%), and intermediate uveitis (2.2\%). Sarcoidosis occurred in 153 patients $(8.4 \%)$, VogtKoyanagi-Harada disease (VKH) in $83(4.6 \%)$, and Behçet's disease in $68(3.7 \%)$. Sarcoidosis peaked in two age groups: $30 \mathrm{~s}$ and $50-70$ s. Of the 1815 patients, 389 eyes of 271 patients $(14.9 \%)$ had cataract surgery, 162 eyes of $133(7.3 \%)$ had vitrectomy, and 124 eyes of $103(5.7 \%)$ had glaucoma surgery. Among cataract surgery patients, 49 (18.1\%) had sarcoidosis, 14 (5.2\%) had VKH, and seven (2.6\%) had Behçet's disease, and visual acuity (VA) was improved in 321 eyes (82.5\%). Among vitrectomy patients, 15 (11.3\%) had acute retinal necrosis, $14(10.5 \%)$ had sarcoidosis, $12(9.0 \%)$ had fungal endophthalmitis, and 11 (8.3\%) had malignant lymphoma (ML); 83 eyes (51.2\%) needed vitrectomy due to vitreous opacity, and VA improved in 88 eyes $(54.3 \%)$. Among glaucoma surgery patients, 13 $(12.6 \%)$ had sarcoidosis, and nine $(8.7 \%)$ had Posner-Schlossman syndrome.

Conclusion: Anterior uveitis was the commonest form of uveitis and sarcoidosis was the commonest underlying disease. The age distribution of sarcoidosis was older than in previous reports. $10 \%$ of patients with sarcoidosis needed glaucoma surgery, and vitrectomy was required in $50 \%$ for vitreous opacity caused by ML or sarcoidosis.

Keywords: anterior uveitis, sarcoidosis, cataract surgery, glaucoma surgery, vitrectomy

\section{Introduction}

Some cases of uveitis are still difficult to diagnose or manage. Intraocular inflammation is influenced by ethnic, geographic, environmental, and lifestyle factors, ${ }^{1}$ and the degree of severity of uveitis varies from mild to severe, with some patients needing long-term follow-up. Epidemiology research is important and helpful for the management of patients with uveitis, ${ }^{2}$ but epidemiological information changes over time. Some papers have previously reported the clinical features of Japanese patients with uveitis, ${ }^{1-7}$ but there have been no reports from Osaka, Japan, in the past two decades.

During long-term follow-up, some patients with uveitis require surgeries, such as cataract surgery, vitrectomy, and glaucoma surgery. Cataract is a frequent 
complication of uveitis and is a result of both the primary disease process with aging and its treatment with corticosteroids, ${ }^{8}$ so cataract surgery is often required for patients with uveitis. Pars plana vitrectomy offers a surgical means to clear vitreous opacities and repair structural complications ${ }^{9,10}$ and microincision vitreous surgery has a safe therapeutic role, even for patients with current uveitis. ${ }^{11}$ Glaucoma surgery is performed to reduce the intraocular pressure (IOP) in uveitis-associated glaucoma and the presence of glaucoma in patients with chronic inflammation of uveitis is associated with an increased risk of visual loss. ${ }^{12}$ In the present study, we investigated the clinical features of patients with ocular inflammation in Osaka, Japan, over the course of 20 years and the related surgical procedures of cataract surgery, vitrectomy, and glaucoma surgery.

\section{Methods}

The Institutional Review Board (IRB) at the Osaka Medical College Hospital approved the current study (Registration No. 2778), and the research adhered to the tenets of the Declaration of Helsinki.

For this retrospective study, we reviewed medical records from the Department of Ophthalmology at Osaka Medical College Hospital for 2730 eyes of 1815 patients with ocular inflammation from April 1999 to March 2019, to evaluate the prevalence of patients with ocular inflammation. Patient consent to review their medical records was not required by the IRB at the Osaka Medical College Hospital. For a definitive diagnosis, common diagnostic criteria reported in the literature were used. ${ }^{1-7}$ Sarcoidosis was diagnosed according to the criteria established by the Japanese Society of Sarcoidosis and Other Granulomatous Disorders. For Behçet's disease, we used the criteria based on the Behçet's Disease Research Committee of Japan. For Vogt-Koyanagi-Harada disease (VKH), we diagnosed according to previously reported criteria. ${ }^{2}$ Other uveitis such as Herpetic iritis, acute retinal necrosis, diabetic iritis, etc. was also diagnosed according to the previous reports. ${ }^{1-7}$ When impossible, we determined as unclassified intraocular inflammation.

At the initial visit, each patient's medical history was obtained through a medical interview. Each patient underwent a comprehensive ophthalmic examination that included the measurement of their best-corrected visual acuity (VA) using a Landolt chart, a measurement of their IOP by Goldmann applanation tonometer, a gonioscopy, and determination of their optic disc and retinal findings using fundus biomicroscopy with a noncontact lens. An additional fluorescein angiography was performed if deemed necessary.

All values are presented as the mean \pm standard deviation. For statistical analysis, the VA that was measured with a Landolt chart was converted to the logarithm of the minimum angle of resolution $(\log$ MAR). To enter all the data, Microsoft Excel was used, and StatView software was used for analyzing data. Statistical testing, when conducted, used a significance level of 0.05 .

\section{Results}

In this retrospective study, we report 2730 eyes of 1815 patients with ocular inflammation examined at the Osaka Medical College Hospital from April 1999 to March 2019. The study included 843 males (mean age $55.5 \pm 17.9$ years) and 972 females (mean age $57.0 \pm 18.9$ years). A malefemale ratio was $0.87: 1$. The patients' mean age was $56.3 \pm$ 18.5 years with the largest age group in their 60s (Figure 1). The mean follow-up period was $40.7 \pm 51.7$ months.

Table 1 shows the diagnostic frequencies of patients with ocular inflammation. Sarcoidosis occurred in 153 patients (8.4\%), VKH in 83 (4.6\%), and Behçet's disease in 68 (3.7\%). Sarcoidosis mainly occurred in two age groups 30 s and $50-70$ s - and was common in elderly women (Figure 2A). By gender, male sarcoidosis was common in the 30 s age group, while female sarcoidosis mainly occurred in the 60-70s age group. VKH mainly occurred in the 30 50 s age group (Figure 2B), while Behçet's disease peaked in two age groups - 20s and 40s (Figure 2C); unclassified intraocular inflammation was observed in 696 patients (38.3\%). Comparing with the diagnostic frequency of new patients in 1999-2003, 2004-2008, 2009-2013, and 2014-2019, the prevalence of patients with Behçet's disease

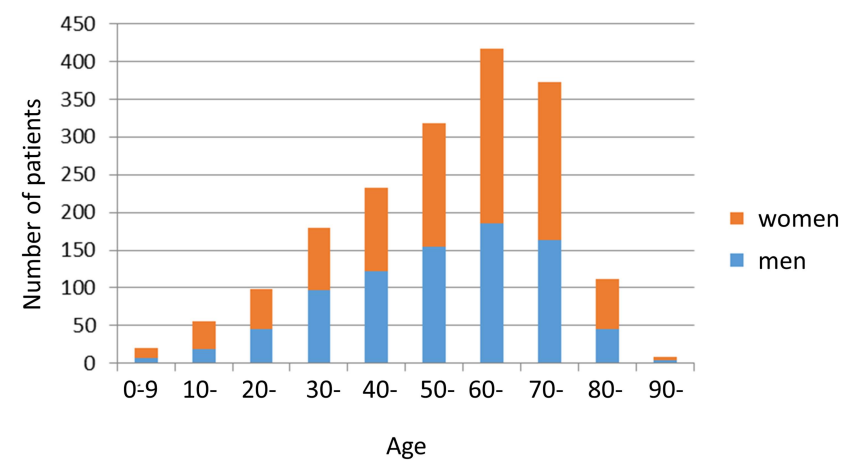

Figure I Distribution of 1815 patients with uveitis from 1999 to 2019 by age and sex. The male-female ratio was $0.87: 1$, the patients' mean age was $56.3 \pm 18.5$ years, and the most common age group was patients in their $60 \mathrm{~s}$. 
Table I The Diagnostic Frequencies of All 1815 Patients with Ocular Inflammation

\begin{tabular}{|c|c|c|c|c|}
\hline Disease & No. of Patients & Men & Women & $\%$ \\
\hline Sarcoidosis & 153 & 53 & 100 & $8.4 \%$ \\
\hline Scleritis & $|4|$ & 81 & 60 & $7.8 \%$ \\
\hline Acute anterior uveitis & 120 & 70 & 50 & $6.6 \%$ \\
\hline Herpetic iritis & 109 & 60 & 49 & $6.0 \%$ \\
\hline Vogt-Koyanagi-Harada disease & 83 & 36 & 47 & $4.6 \%$ \\
\hline Posener-Schlossman syndrome & 68 & 35 & 33 & $3.7 \%$ \\
\hline Behcet's disease & 68 & 36 & 32 & $3.7 \%$ \\
\hline Diabetic iritis & 60 & 36 & 24 & $3.3 \%$ \\
\hline Human $T$ cell lymphotropic virus type-I-associated uveitis & 24 & 6 & 18 & $1.3 \%$ \\
\hline Acute retinal necrosis & 21 & 8 & 13 & $1.2 \%$ \\
\hline Uveitis associated with other systemic diseases & 22 & 8 & 14 & $1.2 \%$ \\
\hline Lens-induced uveitis & 20 & 14 & 6 & $1.1 \%$ \\
\hline Malignant lymphoma & 20 & 11 & 9 & $1.1 \%$ \\
\hline Fungal endophthalmitis & 25 & 15 & 10 & $1.4 \%$ \\
\hline Retinal Vasculitis & 18 & 10 & 8 & $1.0 \%$ \\
\hline Ocular toxoplasmosis & 17 & 12 & 5 & $0.9 \%$ \\
\hline Cytomegalovirus retinitis & 15 & 7 & 8 & $0.8 \%$ \\
\hline Fuchs' heterochromic iridocyclitis & 15 & 5 & 10 & $0.8 \%$ \\
\hline Inflammatory bowel disease-associated uveitis & 14 & 9 & 5 & $0.8 \%$ \\
\hline Tubulointerstitial nephritis and uveitis syndrome-associated uveitis & 10 & 2 & 8 & $0.6 \%$ \\
\hline Punctate inner choroidopathy & 9 & I & 8 & $0.5 \%$ \\
\hline Bacterial endophthalmitis & 12 & 7 & 5 & $0.7 \%$ \\
\hline Ocular toxocariasis & 9 & 2 & 7 & $0.5 \%$ \\
\hline Juvenile idiopathic arthritis-associated uveitis & 9 & 2 & 7 & $0.5 \%$ \\
\hline Syphilis-associated uveitis & 8 & 3 & 5 & $0.4 \%$ \\
\hline Geographic chorioretinopathy & 8 & 4 & 4 & $0.4 \%$ \\
\hline Sympathetic ophthalmia & 8 & 5 & 3 & $0.4 \%$ \\
\hline Multiple evanescent white syndrome & 6 & 1 & 5 & $0.3 \%$ \\
\hline Ocular tuberculosis & 7 & 2 & 5 & $0.4 \%$ \\
\hline Acute posterior multifocal placoid pigment epitheliopathy & 2 & 0 & 2 & $0.1 \%$ \\
\hline Others & 18 & 5 & 13 & $1.0 \%$ \\
\hline \multirow[t]{2}{*}{ Unclassified intraocular inflammation } & 696 & 297 & 399 & $38.3 \%$ \\
\hline & 1815 & 843 & 972 & $100.0 \%$ \\
\hline
\end{tabular}

decreased from $7.6 \%$ to less than $2.0 \%$ with the changing times, but the prevalence of patients with herpetic iritis increased from $5.4 \%$ to $6.6 \%$.

Looking at the distribution of anatomical classification, anterior uveitis was the most common (51.2\%), followed by panuveitis (37.2\%), posterior uveitis (9.4\%), and intermediate uveitis (2.2\%) (Figure 3$)$. The prevalence of scleritis was 141 patients (only two patients, three eyes were posterior scleritis and the others were anterior scleritis) $(7.8 \%)$ after sarcoidosis (8.4\%), acute anterior uveitis was 120 patients (6.6\%), and herpetic iritis was 109 patients $(6.0 \%)$ (Table 1$)$. For the 696 patients with unclassified uveitis, anterior uveitis was found in $53.7 \%$ and panuveitis in $36.8 \%$.

In the entire sample, 389 eyes of 271 patients (14.9\%) had cataract surgery, 162 eyes of $133(7.3 \%)$ had vitrectomy, and
124 eyes of $103(5.7 \%)$ had glaucoma surgery. Cataract surgery was performed on 389 eyes of 271 subjects (156 eyes of 119 males and 233 eyes of 152 females); the mean age was $66.9 \pm 14.2$ years, with a mean observation period of $50.6 \pm 48.1$ months, ranging from 3 to 264 months. By anatomical classification, panuveitis was found in $47.2 \%$, anterior uveitis in $46.9 \%$, posterior uveitis in $4.1 \%$ and intermediate uveitis in $1.8 \%$ of subjects receiving cataract surgery. The period for which the uveitis was quiet preoperatively was $13.5 \pm 28.3$ months, ranging from 0 to 229 months. Of the cataract surgery subjects, $49(18.1 \%)$ had sarcoidosis, 14 (5.2\%) had VKH, 11 (4.1\%) had scleritis (only one subject had posterior scleritis), and seven (2.6\%) had Behçet's disease (Table 2). The intraocular lens was implanted in every patient. Their VA was improved, on 
A
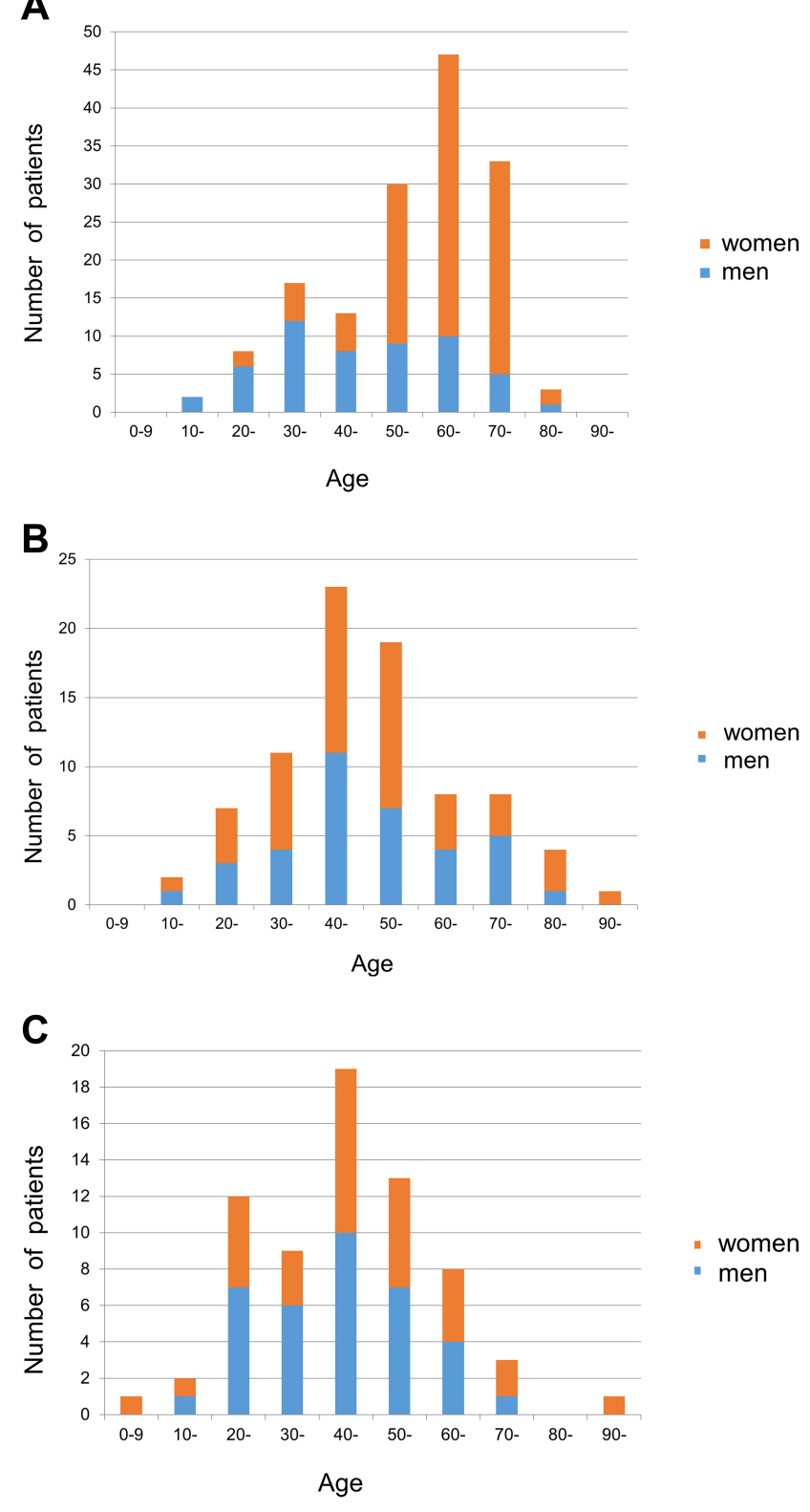

Figure 2 Distribution of patients with sarcoidosis (A), Vogt-Koyanagi-Harada disease (VKH) (B), and Behçet's disease (C). (A) Sarcoidosis mainly occurred in two age groups - 50-70s and 30s - and was common in elderly women. By gender, male sarcoidosis was common in the 30 s age group, while female sarcoidosis mainly occurred in 60 s age group. (B) VKH mainly occurred in the $30-50$ s age group. (C) Behçet's disease peaked in two age groups -40 s and 20 s.

average, from 0.904 to $0.277(\mathrm{P}<0.001$, paired $t$-test $)$ in 318 eyes $(81.7 \%)$. Recurrence within 3 months after cataract surgery was observed in 52 eyes (13.4\%), and occurred more often in cases in which uveitis was quiet for less than 3 months preoperatively ( 72 out of 167 eyes, $43.1 \%$ ) than in cases that were quiet for more than 3 months (46 out of 222 eyes, $20.7 \%$; $\mathrm{P}<0.001$, chi-squared test). Recurrence within 6 months was observed in 80 eyes (20.6\%), among which the mean duration of uveitis being quiet preoperatively was $6.5 \pm$

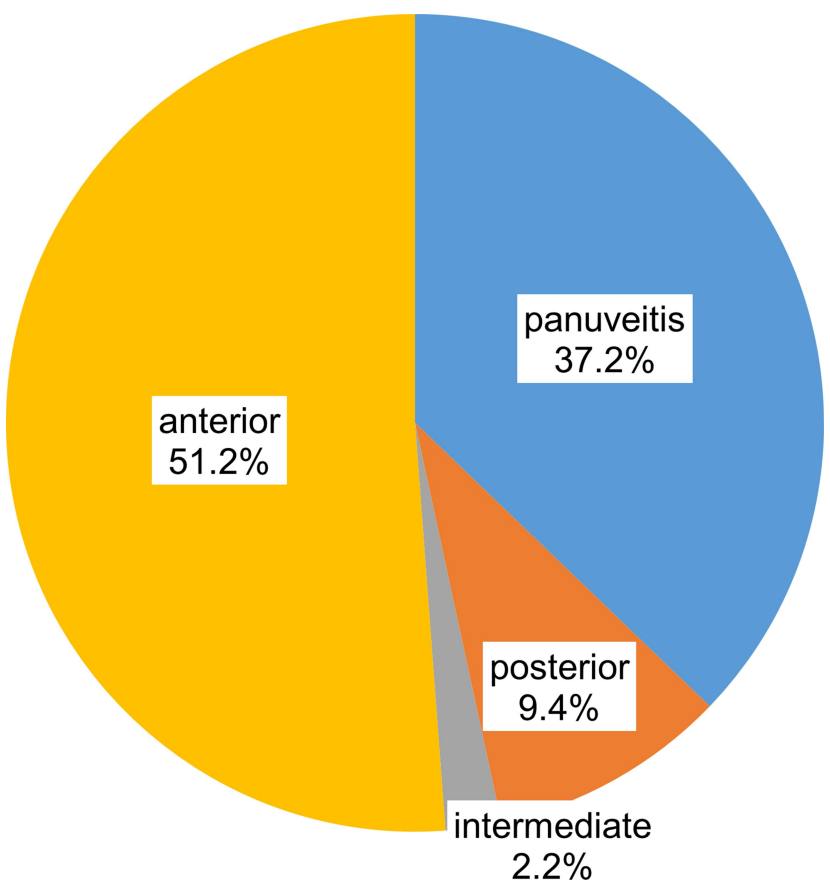

Figure 3 Distribution of 1815 patients with uveitis by anatomical classification. Anterior uveitis was the most common (5I.2\%), followed by panuveitis (37.2\%), posterior uveitis $(9.4 \%)$, and intermediate uveitis $(2.2 \%)$.

19.6 months, which was significantly shorter than in eyes without recurrence within 6 months $(16.6 \pm 30.9$ months; $\mathrm{P}<0.01$, Mann-Whitney $U$-test). Among the recurrent 80 eyes, 41 eyes showed the inflammation of anterior segment only, so that they were treated with eye drops of steroids. Thirty-two eyes with cystoid macular edema and vitreous opacity were treated with sub-Tenon's injection of triamcinolone. Oral prednisolone usage was commenced or increased in dosage in the rest of 7 eyes. The preoperative formation of posterior synechiae of iris was observed in 45 eyes with recurrence within 6 months (38.1\%) compared with 70 eyes without recurrence within 6 months $(25.8 \%$; $\mathrm{P}=0.02$, chi-squared test). The causes of the recurrence occurred within 3 months were sarcoidosis (eight eyes, $15.4 \%$ ), herpetic iritis (four eyes, 7.7\%), VKH (three eyes, $5.8 \%$ ), and Behçet's disease (three eyes, 5.8\%).

Vitrectomy was performed in 162 eyes of 133 subjects ( 75 eyes of 65 males and 87 eyes of 68 females); the mean age was $62.2 \pm 14.1$ years, with a mean observation period of $62.7 \pm 61.1$ months, ranging from 0 to 225 months. By anatomical classification, panuveitis was found in $88.7 \%$, posterior uveitis in $6.8 \%$, intermediate uveitis in $3.8 \%$ and anterior uveitis in $0.7 \%$ of subjects receiving vitrectomy. Fifteen subjects (11.3\%) had acute retinal necrosis (ARN), $14(10.5 \%)$ had sarcoidosis, $12(9.0 \%)$ had fungal 
Table 2 The Diagnostic Frequencies of Patients Receiving Cataract Surgery

\begin{tabular}{|l|l|l|}
\hline Disease & $\begin{array}{l}\text { No. of } \\
\text { Patients }\end{array}$ & $\%$ \\
\hline Sarcoidosis & 49 & $18.1 \%$ \\
Vogt-Koyanagi-Harada disease & 14 & $5.2 \%$ \\
Herpetic iritis & 12 & $4.4 \%$ \\
Scleritis & 11 & $4.1 \%$ \\
Fuchs' heterochromic iridocyclitis & 9 & $3.3 \%$ \\
Posener-Schlossman syndrome & 8 & $3.0 \%$ \\
Diabetic iritis & 8 & $3.0 \%$ \\
Behcet's disease & 7 & $2.6 \%$ \\
Acute anterior uveitis & 5 & $1.8 \%$ \\
Cytomegalovirus anterior uveitis & 5 & $1.8 \%$ \\
Human T cell lymphotropic virus type- & 2 & $0.7 \%$ \\
I-associated uveitis & & \\
Juvenile idiopathic arthritis-associated uveitis & 2 & $0.7 \%$ \\
Inflammatory bowel disease-associated uveitis & 2 & $0.7 \%$ \\
Sympathetic ophthalmia & 2 & $0.7 \%$ \\
Syphilis-associated uveitis & 2 & $0.7 \%$ \\
Rheumatoid arthritis-associated uveitis & 2 & $0.7 \%$ \\
Others & 12 & $4.4 \%$ \\
Unclassified intraocular inflammation & 119 & $43.9 \%$ \\
& 271 & $100.0 \%$ \\
\hline
\end{tabular}

Table 3 The Diagnostic Frequencies of Patients Receiving Vitrectomy

\begin{tabular}{|l|l|l|}
\hline Disease & $\begin{array}{l}\text { No. of } \\
\text { Patients }\end{array}$ & $\%$ \\
\hline Acute retinal necrosis & 15 & $11.3 \%$ \\
Sarcoidosis & 14 & $10.5 \%$ \\
Fungal endophthalmitis & 12 & $9.0 \%$ \\
Malignant lymphoma & 11 & $8.3 \%$ \\
Lens-induced uveitis & 7 & $5.3 \%$ \\
Bacterial endophthalmitis & 6 & $4.5 \%$ \\
Diabetic iritis & 6 & $4.5 \%$ \\
Behcet's disease & 4 & $3.0 \%$ \\
Cytomegalovirus retinitis & 3 & $2.3 \%$ \\
Retinal Vasculitis & 3 & $2.3 \%$ \\
Syphilis-associated uveitis & 2 & $1.5 \%$ \\
Ocular toxoplasmosis & 2 & $1.5 \%$ \\
Human T cell lymphotropic virus type- & 2 & $1.5 \%$ \\
I-associated uveitis & & \\
Acute anterior uveitis & 2 & $1.5 \%$ \\
Others & 4 & $3.0 \%$ \\
Unclassified intraocular inflammation & 40 & $30.1 \%$ \\
& 133 & \\
\hline
\end{tabular}

endophthalmitis, $11(8.3 \%)$ had malignant lymphoma (Table $3)$. Vitrectomy due to vitreous opacity was needed in 83 eyes (51.2\%), and VA was improved in 88 eyes (54.3\%). The causes of vitreous opacity were malignant lymphoma in 18 eyes and sarcoidosis in 15 eyes, etc. The mean VA was significantly improved from 1.085 to 0.711 at the final visit ( $\mathrm{P}<0.001$, paired $t$-test), but 23 eyes $(14.2 \%)$ required reoperation, with the following diagnostic frequencies; five eyes with fungal endophthalmitis, two eyes with sarcoidosis, two eyes with ARN, two eyes with ocular toxoplasmosis, one eye with malignant lymphoma, one eye with cytomegalovirus retinitis, one eye with diabetic iritis (anterior iritis in setting of diabetic vasculopathy or autonomic neuropathy), and nine unclassified. Of the 23 eyes requiring reoperation, 14 had retinal detachment.

Secondary glaucoma developed in $678(37.4 \%)$ of the 1815 patients with uveitis; 75 (11.1\%) patients had sarcoidosis, 61 (9.0\%) had Posner-Schlossman syndrome, and 54 (8.0\%) each had scleritis (all were anterior scleritis.) or herpetic iritis. Steroid-induced glaucoma was found in 55 patients; nine (16.4\%) patients had scleritis, six (10.9\%) had acute anterior uveitis, five (9.1\%) had sarcoidosis, and three (5.5\%) each had VKH and Behçet's disease. Glaucoma surgery was performed on 124 eyes of 103 subjects (64 eyes of 53 males and 60 eyes of 50 females); the mean age was 61.9 \pm 17.7 years, with a mean observation period of $58.5 \pm 48.5$ months, ranging from 2 to 231 months. By anatomical classification, anterior uveitis was found in $63.1 \%$ and panuveitis in $36.9 \%$ of subjects receiving glaucoma surgery. Thirteen subjects $(12.6 \%)$ had sarcoidosis, nine $(8.7 \%)$ had PosnerSchlossman syndrome, six $(5.8 \%)$ had cytomegalovirus infection, and four had scleritis (3.9\%) (Table 4). Trabeculectomy was performed for 104 eyes, trabeculotomy for 14 eyes, peripheral iridectomy for three eyes, and implants of Baerveldt glaucoma drainage devices or Ahmed glaucoma valves for three eyes. After trabeculectomy, IOP significantly decreased from a mean of $37.2 \mathrm{mmHg}$ to $12.9 \mathrm{mmHg}(\mathrm{P}<0.001$, Dunnett). Also in the other glaucoma surgery group, IOP significantly decreased from 33.7 $\mathrm{mmHg}$ to $15.3 \mathrm{mmHg}(\mathrm{P}<0.001$, Dunnett). The IOP was maintained for 72 months after the glaucoma surgery. One month after the surgery, the number of glaucoma medications was significantly reduced from $4.6 \pm 1.3$ to $0.1 \pm 0.4$ in the trabeculectomy group, and from $4.5 \pm 1.7$ to $0.6 \pm 0.8$ in the other glaucoma surgery group, and they were maintained for 72 months after the glaucoma surgery $(\mathrm{P}<0.001$, Dunnett).

\section{Discussion}

In this retrospective study, clinical characteristics of patients with ocular inflammation over the course of 20 years were reviewed and the efficacy of three kinds of 
Table 4 The Diagnostic Frequencies of Patients Receiving Glaucoma Surgery

\begin{tabular}{|l|l|l|}
\hline Disease & $\begin{array}{l}\text { No. of } \\
\text { Patients }\end{array}$ & $\%$ \\
\hline Sarcoidosis & 13 & $12.6 \%$ \\
Posener-Schlossman syndrome & 9 & $8.7 \%$ \\
Cytomegalovirus anterior uveitis & 6 & $5.8 \%$ \\
Scleritis & 4 & $3.9 \%$ \\
Herpetic iritis & 2 & $1.9 \%$ \\
Juvenile idiopathic arthritis-associated uveitis & 2 & $1.9 \%$ \\
Vogt-Koyanagi-Harada disease & $\mathrm{I}$ & $1.0 \%$ \\
Behcet's disease & $\mathrm{I}$ & $1.0 \%$ \\
Human T cell lymphotropic virus type- & $\mathrm{I}$ & $1.0 \%$ \\
I-associated uveitis & & \\
Acute retinal necrosis & $\mathrm{I}$ & $1.0 \%$ \\
Diabetic iritis & $\mathrm{I}$ & $1.0 \%$ \\
Malignant lymphoma & $\mathrm{I}$ & $1.0 \%$ \\
Lens-induced uveitis & $\mathrm{I}$ & $1.0 \%$ \\
Others & 2 & $1.9 \%$ \\
Unclassified intraocular inflammation & 58 & $56.3 \%$ \\
& 103 & \\
\hline
\end{tabular}

surgeries (cataract surgery, vitrectomy, and glaucoma surgery) were investigated. Anterior uveitis such as scleritis, acute anterior uveitis, and herpes iritis, was the most common form of uveitis, and sarcoidosis was the most common underlying disease. The prevalence of Behçet's disease decreased over time, but the prevalence of herpetic iritis increased. Sarcoidosis peaked in two age groups 50-70s and 30s - and was especially common in elderly women, which is consistent with previous reports; ${ }^{1,4}$ however, the sarcoidosis patients seemed to be older in the present study than in previous reports. All surgical procedures for patients with uveitis were mostly effective. In patients with uveitis, the data indicates that cataract surgery should be performed after the suppression of inflammation over 3 months. In subjects undergoing vitrectomy, about $50 \%$ had vitreous opacity, mainly due to malignant lymphoma or sarcoidosis. In patients with sarcoidosis, we found that about $10 \%$ needed glaucoma surgery.

There have already been a number of reports about the epidemiology of uveitis. Researchers from Japan have tended to classify patients with uveitis depending on the diagnosis, such as sarcoidosis, Vogt-Koyanagi-Harada disease (VKH), and Behçet's disease, but uveitis is sometimes difficult to diagnose in real-world clinical practice, and we encountered patients with unclassified uveitis. We determined that unclassified intraocular inflammation was still present in 696 patients (38.3\%) in the current study, so we also considered the distribution of the anatomical classification in the Japanese population with uveitis, and interestingly, we found that anterior uveitis was the most common $(51.2 \%)$, followed by panuveitis $(37.2 \%)$, posterior uveitis (9.4\%), and intermediate uveitis $(2.2 \%)$. Comparing with other Asian countries, ${ }^{13,14}$ recent researches in China found that panuveitis was the most common ${ }^{14}$ and that the frequency of Behçet's disease was higher, which is in contrast with the present study. That study's male-female ratio was similar to the current study, at $0.87: 1$, but the subjects were much younger, at 55.5 years old, and it is well known that the prevalence of uveitis varies by geographic location and that the incidence varies by age and the anatomic location of the inflammatory process. ${ }^{15}$ By attempting anatomical classification, we also noticed that scleritis or acute anterior uveitis were commonly observed in all 1815 patients with ocular inflammation (Table 1) and that the prevalence of patients with herpetic iritis increased over time. This may be because diagnostic polymerase chain reaction (PCR) analysis has become more widespread and PCR of aqueous humor samples easier over time, such as by using a specially designed 30 -gauge needle pipette. ${ }^{16}$

Sarcoidosis has been reported to be the most common uveitis in both the United States and Japan, ${ }^{7}$ as was also found in this current study. Interestingly, our study showed that the age of sarcoidosis patients seemed to be increasing compared with the previous reports, which is probably simply because of a more rapidly aging society. As the world's population ages, we should be careful to make earlier diagnoses because detection of the characteristic ocular signs in elderly patients tends to be delayed. ${ }^{17}$

During the long-term follow-up, some patients with uveitis required surgery, such as cataract surgery, vitrectomy, and glaucoma surgery. We found that all these surgical procedures were mostly effective in patients with uveitis. In cataract surgery, VA was improved in $82.5 \%$ of cases, and recurrence was more common in cases where the uveitis was quiet for less than 3 months preoperatively than in cases in which it was quiet for more than 3 months $(\mathrm{P}<0.001)$. Cataract surgery should therefore only be performed after the suppression of inflammation for at least 3 months, and these results are consistent with previous reports. ${ }^{8,18-20}$

For vitrectomy, about $50 \%$ of patients had vitreous opacity, and malignant lymphoma and sarcoidosis seemed to be the underlying diseases, although vitrectomy for inflammatory eyes with vitreous opacity of unknown etiology has been 
reported. ${ }^{21}$ In the present study, the mean VA was significantly improved after vitrectomy, but $14.2 \%$ of patients required the reoperation, which seems a high incidence. In our study, fungal endophthalmitis was observed in 25 patients, which seemed higher. In general, fungal endophthalmitis results in poor visual outcomes and prompt therapy following early diagnosis helps to reduce significant visual loss, ${ }^{22,23}$ but this is probably because diagnosis of fungal endophthalmitis is often delayed and these cases are treated with steroids without being detected in other hospitals - as Sakai et al reported, there are differences in disease frequencies between general eye clinics and university hospitals. $^{24}$ In addition, we found that 14 eyes $(8.6 \%)$ had retinal detachment among 162 eyes operated in this study. Ophthalmologists should be aware of the risk of retinal detachment in eyes with uveitis.

For glaucoma, secondary glaucoma developed in 678 (37.4\%) of the 1815 patients with uveitis, and 75 (11.1\%) patients had sarcoidosis. In contrast, Takahashi reported incidence of secondary glaucoma in patients with uveitis of $19.7 \%$, with sarcoidosis in $34.1 \%,{ }^{25}$ and the incidence of secondary glaucoma in the current study was higher than in previous reports; ${ }^{12,25}$ although the reason is unclear, it may be related to delayed diagnosis and treatment with aging. Among the 124 eyes of 103 subjects who underwent glaucoma surgery, 13 subjects (12.6\%) had sarcoidosis. We thus determined that about $10 \%$ of patients with sarcoidosis needed glaucoma surgery, but the IOP was generally well controlled after surgery.

There were some limitations of this study. It was a 20year retrospective study, so some clinical findings could not be determined from the clinical charts, and unclassified intraocular inflammation was present in more than $30 \%$ of charts. Diagnostic and therapeutic vitrectomy is helpful, but we still need further development of novel tests.

In conclusion, we reviewed clinical characteristics of patients with ocular inflammation in Osaka, Japan, over the course of 20 years and investigated the efficacy of their required surgeries. We found that anterior uveitis was the most common form of uveitis and the prevalence of patients with herpetic iritis increased over time. Sarcoidosis was the most common underlying disease, and interestingly, the distribution of sarcoidosis was in more elderly patients than in previous reports. About $10 \%$ of the patients with sarcoidosis needed glaucoma surgery, and vitrectomy was required in $50 \%$ for vitreous opacity, which was mainly caused by malignant lymphoma or sarcoidosis.

\section{Disclosure}

The authors report no conflicts of interest in this work.

\section{References}

1. Keino H, Nakashima C, Watanabe T, et al. Frequency and clinical features of intraocular inflammation in Tokyo. Clin Exp Ophthalmol. 2009;37(6):595-601.

2. Shirahama S, Kaburaki T, Nakahara H, et al. Epidemiology of uveitis (2013-2015) and changes in the patterns of uveitis (2004-2015) in the central Tokyo area: a retrospective study. BMC Ophthalmol. 2018;18(1):189. doi:10.1186/s12886-018-0871-6

3. Goto H, Mochizuki M, Yamaki K, Kotake S, Usui M, Ohno S. Epidemiological survey of intraocular inflammation in Japan. Jpn J Ophthalmol. 2007;51(1):41-44. doi:10.1007/s10384-006-0383-4

4. Kitamei H, Kitaichi N, Namba K, et al. Clinical features of intraocular inflammation in Hokkaido, Japan. Acta Ophthalmol. 2009;87 (4):424-428. doi:10.1111/j.1755-3768.2008.01282.x

5. Ohguro N, Sonoda KH, Takeuchi M, Matsumura M, Mochizuki M. The 2009 prospective multi-center epidemiologic survey of uveitis in Japan. Jpn J Ophthalmol. 2012;56(5):432-435. doi:10.1007/s10384012-0158-z

6. Iwata D, Mizuuchi K, Aoki K, et al. Serial frequencies and clinical features of uveitis in Hokkaido, Japan. Ocul Immunol Inflamm. 2017;25(sup1):S15-s18. doi:10.1080/09273948.2016.1184286

7. Kunimi K, Usui Y, Tsubota K, et al. Changes in etiology of uveitis in a single center in Japan. Ocul Immunol Inflamm. 2020;1-6.

8. Mehta S, Linton MM, Kempen JH. Outcomes of cataract surgery in patients with uveitis: a systematic review and meta-analysis. $\mathrm{Am}$ J Ophthalmol. 2014;158(4):676-692.e677. doi:10.1016/j.ajo.2014. 06.018

9. Becker M, Davis J. Vitrectomy in the treatment of uveitis. Am $J$ Ophthalmol. 2005;140(6):1096-1105. doi:10.1016/j.ajo.2005.07. 017

10. Bovey EH, Herbort CP. Vitrectomy in the management of uveitis. Ocul Immunol Inflamm. 2000;8(4):285-291. doi:10.1076/ocii.8.4.28 5.6456

11. Bansal R, Gupta A, Gupta V, et al. Safety and outcome of microincision vitreous surgery in uveitis. Ocul Immunol Inflamm. 2017;25 (6):775-784. doi:10.3109/09273948.2016.1165259

12. Neri P, Azuara-Blanco A, Forrester JV. Incidence of glaucoma in patients with uveitis. J Glaucoma. 2004;13(6):461-465. doi:10.1097/ 01.ijg.0000146391.77618.d0

13. Yang P, Zhong Z, Du L, et al. Prevalence and clinical features of systemic diseases in Chinese patients with uveitis. $\mathrm{Br} J$ Ophthalmol. 2020;bjophthalmol-2020-315960. doi:10.1136/bjophthalmol-2020-3 15960

14. Hao T, Yang LI, Li B, Chen X, Li D, Liu X. Epidemiology of 2000 Chinese uveitis patients from Northeast China. Br J Ophthalmol. 2020;bjophthalmol-2020-316256. doi:10.1136/bjophthalmol-2020316256

15. Tsirouki T, Dastiridou A, Symeonidis C, et al. A focus on the epidemiology of uveitis. Ocul Immunol Inflamm. 2018;26(1):2-16. doi:10.1080/09273948.2016.1196713

16. Kitazawa K, Sotozono C, Koizumi N, et al. Safety of anterior chamber paracentesis using a 30-gauge needle integrated with a specially designed disposable pipette. Br J Ophthalmol. 2017;101(5):548-550. doi:10.1136/bjophthalmol-2016-309650

17. Takayama K, Harimoto K, Sato T, et al. Age-related differences in the clinical features of ocular sarcoidosis. PLoS One. 2018;13(8): e0202585. doi:10.1371/journal.pone.0202585

18. Pålsson S, Andersson Grönlund M, Skiljic D, Zetterberg M. Phacoemulsification with primary implantation of an intraocular lens in patients with uveitis. Clin Ophthalmol. 2017;11:1549-1555. doi:10.2147/OPTH.S143886 
19. Ozates S, Berker N, Cakar Ozdal P, Ozdamar Erol Y. Phacoemulsification in patients with uveitis: long-term outcomes. BMC Ophthalmol. 2020;20(1):109. doi:10.1186/s12886-020-01373-5

20. Pistilli M, Joffe MM, Gangaputra SS, et al. Visual acuity outcome over time in non-infectious uveitis. Ocul Immunol Inflamm. 2019;1-8. doi:10.1080/09273948.2019.1687733

21. Sato T, Kinoshita R, Taguchi M, et al. Assessment of diagnostic and therapeutic vitrectomy for vitreous opacity associated with uveitis with various etiologies. Medicine. 2018;97(2):e9491. doi:10.1097/ MD.0000000000009491

22. Celiker H, Kazokoglu H. The role of pars plana vitrectomy in the management of fungal endogenous endophthalmitis. Eur J Ophthalmol. 2020;30(1):88-93. doi:10.1177/1120672118815105
23. Chhablani J. Fungal endophthalmitis. Expert Rev Anti Infect Ther. 2011;9(12):1191-1201. doi:10.1586/eri.11.139

24. Sakai JI, Usui Y, Sakai M, Yokoi H, Goto H. Clinical statistics of endogenous uveitis: comparison between general eye clinic and university hospital. Int Ophthalmol. 2010;30(3):297-301. doi:10.1007/ s10792-009-9336-5

25. Takahashi T, Ohtani S, Miyata K, Miyata N, Shirato S, Mochizuki M. A clinical evaluation of uveitis-associated secondary glaucoma. Jpn $J$ Ophthalmol. 2002;46(5):556-562. doi:10.1016/S0021-5155(02) 00549-X
Clinical Ophthalmology

\section{Publish your work in this journal}

Clinical Ophthalmology is an international, peer-reviewed journal covering all subspecialties within ophthalmology. Key topics include: Optometry; Visual science; Pharmacology and drug therapy in eye diseases; Basic Sciences; Primary and Secondary eye care; Patient Safety and Quality of Care Improvements. This journal is indexed on PubMed

Submit your manuscript here: https://www.dovepress.com/clinical-ophthalmology-journa
Dovepress

Central and CAS, and is the official journal of The Society of Clinical Ophthalmology (SCO). The manuscript management system is completely online and includes a very quick and fair peer-review system, which is all easy to use. Visit http://www.dovepress.com/ testimonials.php to read real quotes from published authors. 